\title{
IMPLEMENTACIÓN DE UNA METODOLOGÍA ANALÍTICA PARA LA CUANTIFICACIÓN DE PROTEÍNAS EN LA MICROALGA Arthrospira platensis
}

\author{
Leenin Flores Ramos ${ }^{* a}$, Anthony Ruiz Soto ${ }^{\mathrm{a}}$
}

\begin{abstract}
RESUMEN
La metodología analítica implementada para la cuantificación de proteínas en la microalga Arthorspira platensis por espectrofotometría UV-Vis fue el método de Hartree-Lowry. Para lograr este objetivo, se optimizaron los factores de extracción de proteínas por el método estadístico de diseño de superficie de respuesta de Box Behnken y se obtuvieron los siguientes resultados de los parámetros implementados: la linealidad, donde se reportó un coeficiente de determinación $\left(\mathrm{R}^{2}\right)$ mayor a 0,995 , en el estudio de repetibilidad un coeficiente de variación menor al $2 \%$, un porcentaje de recuperación entre $94 \%$ y $101 \%$, los límites de detección y cuantificación fueron $2,36 \mu \mathrm{g} / \mathrm{mL}$ y $7,86 \mu \mathrm{g} / \mathrm{Ml}$, respectivamente y el estudio de la robustez demostró que el método analítico tuvo mayor sensibilidad al cambio del peso de la muestra y al cambio del volumen de reactivo $\mathrm{C}$ respecto al resto de factores evaluados.
\end{abstract}

Palabras clave: Espectrofotometría, microalga, Box Behnken, Youden-Steiner.

\section{IMPLEMENTATION OF ANALYTICAL METHODOLOGY FOR QUANTIFICATION OF PROTEINS IN Arthrospira platensis MICROALGAE}

\begin{abstract}
The Hartree-Lowry method was developed and implemented for quantification of protein in Arthorspira platensis microalgae by spectrophotometry UV-Vis. In order to achieve this goal, the protein extraction factors were optimized by Box Behnken design of response surface and the following results of the implementation parameters were obtained: the linearity showed a coefficient of determination $\left(\mathrm{R}^{2}\right)$ greater than 0.995 , a coefficient of variation less than $2 \%$ for repeatability, a percentage recovery within $94 \%$ to $101 \%$, the limit of detection and limit of quantification were $2.36 \mu \mathrm{g} / \mathrm{mL}$ and $7.86 \mu \mathrm{g} / \mathrm{mL}$ respectively, and the robustness showed that some parameters such as sample weight and volume of reagent $\mathrm{C}$ presented high influence in the evaluated factors.
\end{abstract}

Key words: Spectrophotometry, microalgae, Box Behnken, Youden-Steiner.

\footnotetext{
${ }^{a}$ Laboratorio de Análisis Instrumental - Laboratorio de Biotecnología Acuática, Instituto del Mar del Perú, Esquina Gamarra y General Valle s/n Chucuito Callao, Callao, Perú, lflores@imarpe.gob.pe
} 


\section{INTRODUCCIÓN}

Actualmente, las microalgas han sido ampliamente estudiadas por sus numerosos contenidos de compuestos bioactivos, que son aprovechados para su uso comercial ${ }^{1}$, por ejemplo las microalgas: Dunaliella salina para la producción de $\beta$-caroteno, Haematococcus pluvialis para la producción de Astaxantina, Schizochytrium sp. para la producción de ácido docosahexaenoico (DHA), etc.

En particular la microalga Arthrospira platensis es producida por la industria como suplemento alimenticio en diferentes países como Estados Unidos, China, Cuba, India, Tailandia, México, Chile y Sud África, debido a su alto contenido de proteínas $50-70$ \% y aminoácidos esenciales ${ }^{2}$. La cuantificación de proteínas es esencial no solo para garantizar la calidad e inocuidad de los alimentos, sino también para facilitar su comercio ${ }^{3}$.

Los laboratorios analíticos han desarrollado diversas técnicas para la cuantificación de proteínas, por ejemplo el método normalizado Kjeldahl que se aplica en diferentes matrices como cerveza, leche, pan, etc. ${ }^{4}$ y métodos espectrofotométricos como Lowry, Biuret, Bradford y BCA. Por otro lado, el método de Hartree-Lowry (modificación subsecuente del método de Lowry) es un método espectrofotométrico utilizado para la cuantificación de proteínas debido que presenta ventajas tales como su sensibilidad, límite de detección del orden de 15 $\mu \mathrm{g}$ de proteína, precisión y fácil implementación ${ }^{5}$.

De acuerdo a normas vigentes, las metodologías no normalizadas deben ser validadas (Resolución No 0008-2003/INDECOPI-CRT), por ello, la Asociación de las Comunidades Analíticas (AOAC Internacional), Eurachem, el Codex Alimentarius, Farmacopea de los Estados Unidos de América (USP), ICH, ISO y otros organismos internacionales, recomiendan la implementación del método analítico evaluando los parámetros de desempeño como: veracidad, precisión, especificidad, linealidad, límite de detección, límite de cuantificación, sensibilidad y robustez ${ }^{6}$.

El objetivo del presente trabajo es implementar el método analítico de Hartree-Lowry para la cuantificación de proteínas en la microalga Arthrospira platensis por espectrofotometría UV-Vis.

\section{PARTE EXPERIMENTAL}

\section{Muestra}

La microalga Arthrospira platensis liofilizada fue proveída por la Sala de Procesos Laboratorio de Biotecnología Acuática, IMARPE.

\section{Reactivos}

Albúmina de suero bovino BSA (Sigma-Aldrich®), hidróxido de sodio P.A. (Merck), tartrato 
de sodio y potasio tetrahidratado P.A. (Merck), carbonato de sodio anhidro P.A. (Merck), sulfato de cobre pentahidratado P.A. (Merck), reactivo del fenol según Folin-Ciocalteau (Merck).

\section{Equipos}

Espectrofotómetro UV-Visible Varian Cary 50, Agitador Vórtex ZX Classic VELP Scientífica, balanza analítica Sartorius MSU225S-000-DU, centrífuga refrigerada Eppendorf Centrifuge 5702R, baño de ultrasonido Branson 2510, campana extractora de gases LABCONCO, baño maría MRC BH-200 y baño maría Eyela SB 2000.

\section{Solución patrón de proteínas}

Se pesó 30,0 mg de albúmina de suero bovino, se transfirió a una fiola de $50 \mathrm{~mL}$ y se añadió $25 \mathrm{~mL}$ de agua ultrapura, se sonicó por 5 min y finalmente se enrazo con agua ultrapura para obtener una concentración de $600 \mu \mathrm{g} / \mathrm{mL}$.

\section{Extracción de proteínas}

Se pesaron 5,0 mg de microalga liofilizada y se añadió $5 \mathrm{~mL}$ de hidróxido de sodio, luego se sonicó en baño de ultrasonido por 15 min y se calentó en baño maría para realizar la hidrólisis. Se centrifugó la muestra a $4{ }^{\circ} \mathrm{C}$ por $10 \mathrm{~min}$ a $3500 \mathrm{rpm}$ y se extrajo $200 \mu \mathrm{L}$ del sobrenadante para el análisis de proteínas.

\section{Método analítico}

Las proteínas extraídas mediante hidrólisis alcalina y las soluciones estándares fueron cuantificadas por el método de Hartree-Lowry, el cual consistió en la adición de $0,9 \mathrm{~mL}$ de reactivo A (hidróxido de sodio $0,5 \mathrm{~N}$, carbonato de sodio anhidro $10 \%$ y tartrato de sodio y potasio tetrahidratado $0,2 \%$ ), seguido de una incubación a $50{ }^{\circ} \mathrm{C}$ durante 10 minutos, luego la adición de $0,1 \mathrm{~mL}$ de reactivo $\mathrm{B}$ (hidróxido de sodio $0,1 \mathrm{~N}$, sulfato de cobre pentahidratado $1 \%$ y tartrato de sodio y potasio tetrahidratado $2 \%$ y finalmente la adición de $3 \mathrm{~mL}$ de reactivo $\mathrm{C}$ (reactivo del fenol según Folin-Ciocalteau: Agua 2:15 v/v). Se midió las absorbancias en el espectrofotómetro a una longitud de onda de $650 \mathrm{~nm}$.

\section{Optimización de los parámetros de extracción de proteínas}

El método estadístico utilizado para la optimización fue el diseño de superficies de Box Behnken $^{7}$ y los resultados fueron procesados con el software Minitab 16. Se optimizó la variable respuesta Absorbancia con los siguientes factores, sugeridos por Arredondo y Voltolina ${ }^{8}$, Concentración de hidróxido de sodio $\left(C_{N a O H}\right)$, tiempo de extracción $(t)$ y temperatura $(T)$. Los niveles de los factores se muestran en la tabla 1. Es necesario mencionar que los valores optimizados fueron los usados en todas las pruebas de implementación. 
Tabla 1. Factores y niveles del diseño de superficies

\begin{tabular}{ccc}
\hline $\begin{array}{c}\text { Concentración } \\
\text { NaOH (N) }\end{array}$ & $\begin{array}{c}\text { Tiempo de } \\
\text { extracción }(\mathbf{m i n})\end{array}$ & $\begin{array}{c}\text { Temperatura } \\
\left({ }^{\circ} \mathbf{C}\right)\end{array}$ \\
\hline $0,1(-1)$ & $30(-1)$ & $60(-1)$ \\
$0,5(0)$ & $45(0)$ & $80(0)$ \\
$0,9(+1)$ & $60(+1)$ & $100(+1)$ \\
\hline
\end{tabular}

\section{Parámetros de la implementación}

\section{Linealidad}

Se construyó la curva de calibración por duplicado en el rango de concentración de 30$300 \mu \mathrm{g} / \mathrm{mL}$ usando 10 niveles $(30,60,90,120,150,180,210,240,270$ y $300 \mu \mathrm{g} / \mathrm{mL})$ y empleando la solución patrón albúmina suero de bovino (BSA).

\section{Límite de detección (LOD) y límite de cuantificación (LOQ)}

Estos parámetros se obtuvieron de los valores obtenidos de la pendiente $(\mathrm{m})$ y el error estándar del intercepto $(\mathrm{sb})$ de la curva de calibración de acuerdo a las siguientes fórmulas: $\mathrm{LOD}=3 * \mathrm{sb} / \mathrm{m}$ y LOQ $=10 * \mathrm{sb} / \mathrm{m}$.

\section{Porcentaje de recuperación}

Se evaluó el porcentaje de recuperación usando tres niveles de concentración de fortificación $\left(\mathrm{C}_{\mathrm{a}}\right)$ correspondientes al $25 \%, 50 \%$ y $75 \%$ de la concentración estimada del analito en la muestra $\left(\mathrm{C}_{\mathrm{u}}, 80 \mu \mathrm{g} / \mathrm{mL}\right)$ de acuerdo a la siguiente formula: $\% \mathrm{R}=\left(\left(\mathrm{C}_{\mathrm{f}}-\mathrm{C}_{\mathrm{u}}\right) / \mathrm{C}_{\mathrm{a}}\right) \times 100 \%$ donde $\mathrm{C}_{\mathrm{f}}$ es la concentración del analito en la muestra fortificada.

\section{Repetibilidad}

Se evaluó la repetibilidad analizando nueve muestras independientes bajo las siguientes condiciones: un mismo analista, mismo laboratorio, intervalos de tiempos cortos y los mismos equipos.

\section{Robustez}

Se aplicó la metodología de Youden-Steiner ${ }^{9}$ y se seleccionaron los siguientes factores, de acuerdo al criterio del laboratorio, Peso de muestra: $\mathrm{A}=5 \mathrm{mg}, \mathrm{a}=7 \mathrm{mg}$; Volumen de Reactivo A: $\mathrm{B}=900 \mu \mathrm{L}, \mathrm{b}=1000 \mu \mathrm{L}$; Volumen de Reactivo $B: \mathrm{C}=100 \mu \mathrm{L}, \mathrm{c}=150 \mu \mathrm{L}$; Volumen de Reactivo $C: \mathrm{D}=3 \mathrm{~mL}, \mathrm{~d}=4 \mathrm{~mL}$; Tiempo de incubación luego de añadir Reactivo A: $\mathrm{E}=10$ $\min , \mathrm{e}=5 \mathrm{~min}$; Tiempo de incubación luego de añadir Reactivo $B: \mathrm{F}=0 \mathrm{~min}, \mathrm{f}=10 \mathrm{~min}$ y el Tiempo de lectura: $\mathrm{G}=$ instante, $\mathrm{g}=$ después de $30 \mathrm{~min}$. 
Tabla 2. Prueba de robustez de Youden y Steiner

\begin{tabular}{lcccccccc}
\hline \multicolumn{1}{c}{ Factores } & \multicolumn{7}{c}{ Experimentos } \\
\cline { 2 - 9 } & $\mathbf{1}$ & $\mathbf{2}$ & $\mathbf{3}$ & $\mathbf{4}$ & $\mathbf{5}$ & $\mathbf{6}$ & $\mathbf{7}$ & $\mathbf{8}$ \\
\hline Peso de muestra & A & A & A & A & a & a & a & a \\
Volumen de Reactivo A & B & B & b & b & B & B & b & b \\
Volumen de Reactivo B & C & c & C & c & C & c & C & c \\
Volumen de Reactivo C & D & D & d & d & d & d & D & D \\
Tiempo de incubación luego de & E & e & E & e & e & E & e & E \\
añadir Reactivo A & & & & & & & & \\
Tiempo de incubación luego de & F & f & f & F & F & f & f & F \\
añadir Reactivo B & G & g & g & G & g & G & G & g \\
Tiempo de lectura & S & t & u & v & W & x & y & z \\
\hline Resultados & & & & & & & &
\end{tabular}

Los efectos fueron calculados con la siguiente formula:

$$
E_{x}=\frac{\sum Y(+)}{n / 2}-\frac{\sum Y(-)}{n / 2}
$$

Donde Ex son los efectos del factor $\mathrm{x}, \sum \mathrm{Y}(+)$ es la suma de los resultados del factor $\mathrm{x}$ en el extremo superior del intervalo definido, $\sum \mathrm{Y}(-)$ es la suma de los resultados del factor $\mathrm{x}$ en el extremo inferior del intervalo definido y n es el número total de experimentos.

\section{RESULTADOS Y DISCUSIÓN}

\section{Optimización de los parámetros de extracción de proteínas:}

Los resultados del diseño de superficies se muestran en la tabla 3.

Tabla 3. Resultados de la matriz del diseño de superficies

\begin{tabular}{ccccc}
\hline Experimento & $\begin{array}{c}\text { Concentración } \\
\text { de NaOH }\end{array}$ & $\begin{array}{c}\text { Tiempo de } \\
\text { extracción }\end{array}$ & Temperatura & Absorbancia \\
\hline 12 & 0 & +1 & +1 & 0,3187 \\
5 & -1 & 0 & -1 & 0,2333 \\
13 & 0 & 0 & 0 & 0,3016 \\
15 & 0 & 0 & 0 & 0,3278 \\
1 & -1 & -1 & 0 & 0,2430 \\
6 & +1 & 0 & -1 & 0,2334 \\
4 & +1 & +1 & 0 & 0,2733 \\
7 & -1 & 0 & +1 & 0,2568 \\
8 & +1 & 0 & +1 & 0,2240 \\
10 & 0 & +1 & -1 & 0,3491 \\
11 & 0 & -1 & +1 & 0,2896 \\
14 & 0 & 0 & 0 & 0,3336 \\
3 & -1 & +1 & 0 & 0,2594 \\
2 & +1 & -1 & 0 & 0,2462 \\
9 & 0 & -1 & -1 & 0,3267 \\
\hline
\end{tabular}


Al graficar la Absorbancia vs la Concentración de NaOH y la Temperatura se puede apreciar una tendencia cuadrática en la superficie debido a la curvatura formada en los niveles del factor concentración $\mathrm{NaOH}$.

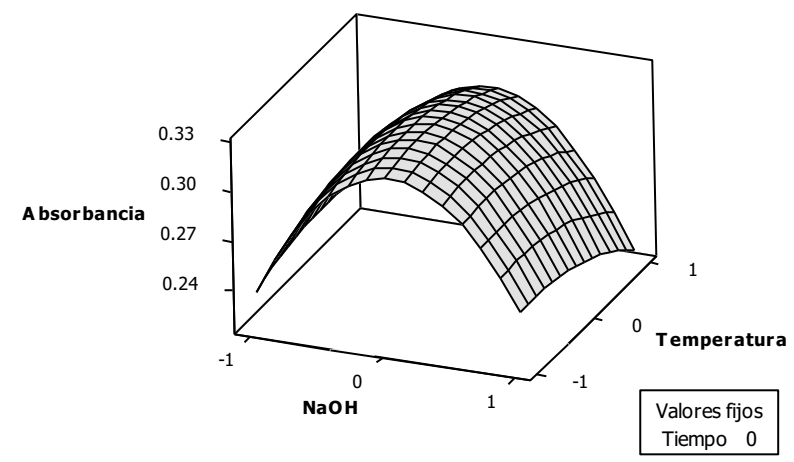

Figura 1. Gráfica de superficies de la Absorbancia vs Temperatura, $\mathrm{NaOH}$

El análisis de varianza del diseño de superficies (tabla 4) confirma que el modelo cuadrático es significativo ( $p$-valor $=0,003$ ) para un nivel de significancia $(\alpha)$ igual a 0,05 . Los efectos de las interacciones y términos lineales no fueron significativas ( $\mathrm{p}$-valor $>\alpha=0,05$ ). De otro lado, los efectos de los términos cuadráticos fueron significativos solo en el caso de la concentración de $\mathrm{NaOH}$ ( $\mathrm{p}$-valor $=0,001<\alpha=0,05)$. La ecuación de regresión resultante fue la siguiente:

$$
\begin{aligned}
& \text { Absorbancia }=0,321-0,002 \mathrm{C}_{\mathrm{NaOH}}+0,012 \mathrm{t}-0,007 \mathrm{~T}+0,003 \mathrm{C}_{\mathrm{NaOH}} \mathrm{t}- \\
& 0,008 C_{\mathrm{NaOH}} \mathrm{T}+0,002 \mathrm{tT}-0,075 \mathrm{C}_{\mathrm{NaOH}}{ }^{2}+0,009 \mathrm{t}^{2}-0,009 \mathrm{~T}^{2}
\end{aligned}
$$

Tabla 4. Análisis de Varianza (ANOVA) del diseño de superficies

\begin{tabular}{lccccc}
\hline \multicolumn{1}{c}{ Fuente } & GL & SC Sec. & SC Ajust. & CM Ajust. & P valor \\
\hline Regresión & 9 & 0,02343 & 0,02343 & 0,00260 & 0,019 \\
Lineal & 3 & 0,00152 & 0,00152 & 0,00051 & 0,332 \\
$\quad \mathrm{NaOH}$ & 1 & 0,00003 & 0,00003 & 0,00003 & 0,779 \\
$\quad$ Tiempo & 1 & 0,00113 & 0,00113 & 0,00113 & 0,131 \\
Temperatura & 1 & 0,00036 & 0,00036 & 0,00036 & 0,357 \\
Cuadrado & 3 & 0,02160 & 0,02160 & 0,00720 & $\mathbf{0 , 0 0 3}$ \\
$\quad$ NaOH*NaOH & 1 & 0,02091 & 0,02068 & 0,02068 & $\mathbf{0 , 0 0 1}$ \\
$\quad$ Tiempo*Tiempo & 1 & 0,00037 & 0,00032 & 0,00032 & 0,38 \\
Temperatura*Temperatura & 1 & 0,00032 & 0,00032 & 0,00032 & 0,382 \\
interacción & 3 & 0,00031 & 0,00031 & 0,00010 & 0,826 \\
$\quad$ NaOH*Tiempo & 1 & 0,00003 & 0,00003 & 0,00003 & 0,785 \\
$\quad \mathrm{NaOH}$ Temperatura & 1 & 0,00027 & 0,00027 & 0,00027 & 0,417 \\
$\quad$ Tiempo*Temperatura & 1 & 0,00001 & 0,00001 & 0,00001 & 0,864 \\
Error residual & 5 & 0,00173 & 0,00173 & 0,00035 & \\
Falta de ajuste & 3 & 0,00115 & 0,00115 & 0,00038 & 0,459 \\
Error puro & 2 & 0,00058 & 0,00058 & 0,00029 & \\
Total & 14 & 0,02516 & & & \\
\hline
\end{tabular}


Al optimizar la ecuación con el software se obtuvieron las condiciones óptimas de extracción de proteínas (figura 2): concentración de $\mathrm{NaOH}=0,5 \mathrm{~N}[0,0101]$, tiempo $=60 \min [1,0000]$ y temperatura $=75^{\circ} \mathrm{C}[-0,2727]$.

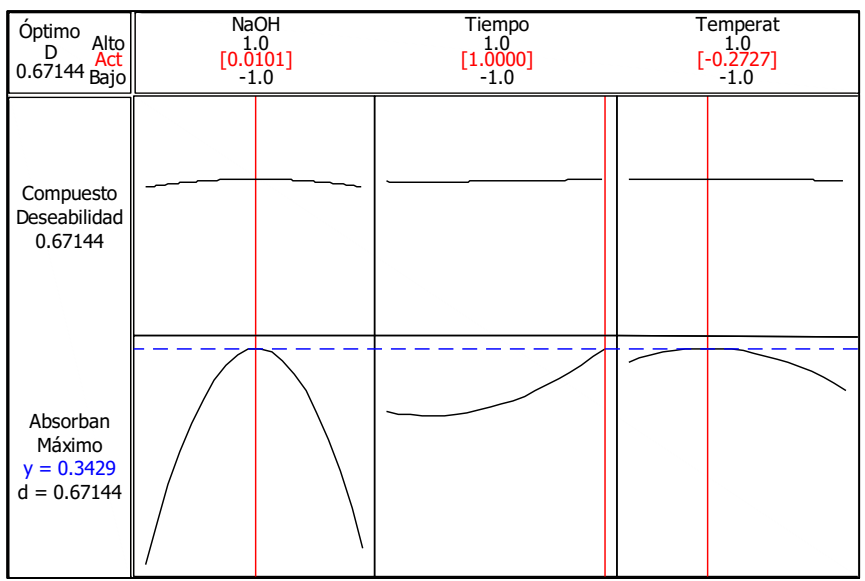

Figura 2. Gráfica de optimización de los factores

\section{Linealidad}

Al graficar los resultados de la prueba de linealidad (figura 3) se observa que la curva de calibración no presenta linealidad en todo su rango experimental (figura 3A), debido a ello se seleccionó el intervalo de trabajo de 30-150 $\mu \mathrm{g} / \mathrm{mL}$ (5 niveles) donde se observa linealidad (figura 3B).
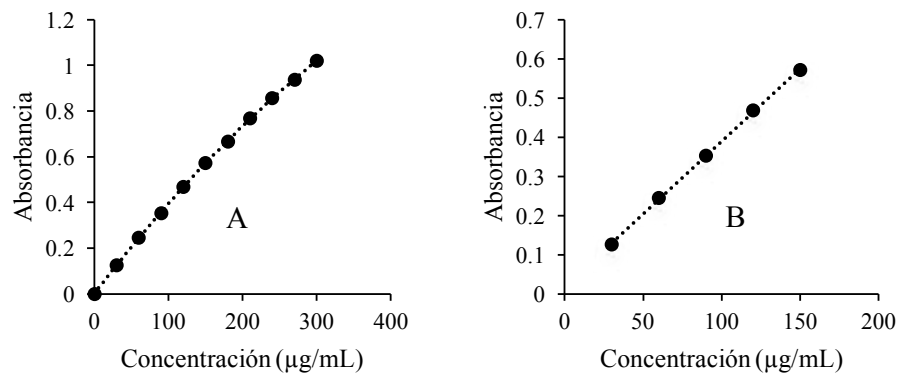

Figura 3. Gráfico de dispersión de la prueba de linealidad 
Para validar si el modelo es de regresión lineal en el intervalo de trabajo de 30-150 $\mu \mathrm{g} / \mathrm{mL}$ se verificaron cuatro supuestos estadísticos:

\section{1.- Supuesto de linealidad}

El coeficiente de determinación $\left(R^{2}\right)$ 0,9995, superó el valor de referencia $R^{2} \geq 0,995$, usualmente aceptados en los laboratorios como criterio de aceptación ${ }^{10,11}$, asegurando el supuesto de linealidad.

\section{2.- Supuesto de independencia de los residuos}

El estadístico de Durbin-Watson presentó un valor de 2,33 cercano al valor referencial 2 indicando independencia o incorreción de los residuos.

\section{3.- Supuesto de residuos constantes (Homocedasticidad)}

La prueba estadística de homocedasticidad contrasta la hipótesis nula de que variable respuesta tiene varianzas iguales en cada nivel del factor. La prueba de Bartlett presentó un $\mathrm{p}$-valor $=0,951>\alpha=0,05$, indicando que las varianzas de los residuos no presentan diferencias significativas, asegurando el supuesto de homocedasticidad.

\section{4.- Supuesto de normalidad de residuos}

La prueba estadística de normalidad contrasta la hipótesis nula de que la variable tiene una distribución normal. El estadístico Kolmogorov - Smirnov presentó un p-valor $=0,090>\alpha=$ 0,05 , por lo tanto los residuos presentan una distribución normal.

La tabla 5 muestra los estadísticos de la regresión del modelo lineal.

Tabla 5. Análisis de regresión de la Absorbancia vs Concentración

\begin{tabular}{|c|c|c|c|c|c|c|}
\hline Fuente & SS & df & MS & \multirow{2}{*}{\multicolumn{2}{|c|}{$\begin{array}{l}\text { Numero de observaciones } \\
\text { ANOVA }\end{array}$}} & \multirow{2}{*}{10} \\
\hline Modelo & 0,24876 & 1 & 0,24876 & & & \\
\hline Residual & 0,00012 & 8 & 0,00001 & \multirow{2}{*}{$\begin{array}{l}\mathrm{F}(1,8) \\
\mathrm{p} \text {-valor }\end{array}$} & & \multirow{2}{*}{$\begin{array}{l}16037,51 \\
\mathbf{0 , 0 0 0 0} \\
0,9995\end{array}$} \\
\hline \multirow[t]{2}{*}{ Total } & 0,24888 & 9 & 0,27653 & & & \\
\hline & Coeficiente & $\begin{array}{c}\text { Error } \\
\text { estándar }\end{array}$ & $\mathbf{t}$ & $P>|t|$ & $\begin{array}{r}\text { (Inte } \\
\text { confian } \\
\end{array}$ & $\begin{array}{l}\text { rvalo de } \\
\text { za al } 95 \% \text { ) }\end{array}$ \\
\hline pendiente & 0,00371 & 0,00003 & 126,64 & 0,000 & 0,00364 & 0,00378 \\
\hline intercepto & 0,01822 & 0,00292 & 6,24 & 0,000 & 0,01149 & 0,02496 \\
\hline
\end{tabular}

El análisis de varianza (ANOVA) de regresión muestra que los coeficientes de regresión son significativamente diferentes de cero ( $\mathrm{p}$-valor $<\alpha=0,05$ ).

El intervalo de confianza al $95 \%$ de la pendiente fue [0,00364 - 0,00378]. Finalmente el modelo de regresión fue:

\section{Absorbancia $=0.01822+0.00371 *[$ Concentración $]$}




\section{Límite de detección (LOD) y límite de cuantificación (LOQ)}

En el rango de trabajo de la curva de calibración de 30 a $150 \mu \mathrm{g} / \mathrm{mL}$, el $\mathrm{s}_{\mathrm{b}}=0,00292$ y el $\mathrm{m}=0,00371$, por lo tanto el $\mathrm{LOD}=2,36 \mu \mathrm{g} / \mathrm{mL}$ y el $\mathrm{LOQ}=7,86 \mu \mathrm{g} / \mathrm{mL}$. Estos valores se encuentran en el orden con lo reportado por Hartree 5 .

\section{Porcentaje de recuperación}

Los resultados (tabla 6) muestran que el promedio de los porcentajes de recuperación para las concentraciones estudiadas es $97,13 \%$ y se encuentra dentro del rango de aceptación ( 90 - $107 \%$ ) de acuerdo al Manual del Codex Alimentarius ${ }^{12}$, por lo que se puede aseverar que el efecto de la matriz no es significativo.

Tabla 6. Valores de porcentaje de recuperación

\begin{tabular}{|c|c|c|c|c|}
\hline $\begin{array}{c}\text { Nivel de } \\
\text { concentración }\end{array}$ & $\begin{array}{c}\text { Concentración } \\
(\mu \mathrm{g} / \mathrm{mL})\end{array}$ & $\begin{array}{c}\text { Promedio } \\
(\mu \mathrm{g} / \mathrm{mL})\end{array}$ & $\begin{array}{c}\text { Recuperación } \\
(\mu \mathrm{g} / \mathrm{mL})\end{array}$ & $\begin{array}{c}\text { Recuperación } \\
(\%)\end{array}$ \\
\hline Muestra & $\begin{array}{l}78,21 \\
81,03 \\
79,92\end{array}$ & 79,72 & 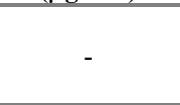 & 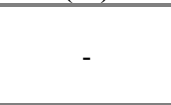 \\
\hline $\begin{array}{l}\text { Nivel } 1 \\
(25 \%)\end{array}$ & $\begin{array}{c}98,37 \\
99,50 \\
101,34\end{array}$ & 99,74 & 20,02 & 100,09 \\
\hline $\begin{array}{l}\text { Nivel } 2 \\
(50 \%)\end{array}$ & $\begin{array}{l}115,58 \\
118,37 \\
119,00\end{array}$ & 117,65 & 37,93 & 94,82 \\
\hline \multirow[t]{2}{*}{$\begin{array}{l}\text { Nivel } 3 \\
(75 \%)\end{array}$} & $\begin{array}{l}136,63 \\
138,37 \\
137,84\end{array}$ & 137,61 & 57,89 & 96,49 \\
\hline & & & Promedio & 97,13 \\
\hline
\end{tabular}

\section{Repetibilidad}

La tabla 7 muestra la precisión bajo condiciones de repetibilidad, el resultado fue un coeficiente de variación $(\mathrm{CV} \%=1,79 \%)$ y se encuentra dentro del límite de referencia establecido 9 (CV \% < 5,3\%).

Tabla 7. Resultados obtenidos de la prueba de repetibilidad

\begin{tabular}{cc}
\hline Réplica & Proteína (\%) \\
\hline 1 & 41,11 \\
2 & 40,26 \\
3 & 40,57 \\
4 & 41,24 \\
5 & 42,22 \\
6 & 40,24 \\
7 & 41,03 \\
8 & 40,91 \\
9 & 39,70 \\
\hline Promedio & 40,81 \\
Desviación Estándar & 0,73 \\
CV \% & $1,79 \%$ \\
\hline
\end{tabular}




\section{Robustez}

La tabla 8 muestra los resultados de los tratamientos de acuerdo al diseño factorial de YoudenSteiner.

Tabla 8. Resultados del diseño factorial de Youden-Steiner

\begin{tabular}{ccccccccc}
\hline Tratamientos & $\mathbf{s}$ & $\mathbf{t}$ & $\mathbf{u}$ & $\mathbf{v}$ & $\mathbf{w}$ & $\mathbf{x}$ & $\mathbf{y}$ & $\mathbf{z}$ \\
Proteínas \% & 39,34 & 43,48 & 28,35 & 28,06 & 20,14 & 19,35 & 26,30 & 26,39 \\
\hline
\end{tabular}

La figura 4 muestra la proporción de los efectos, donde los factores más sensibles del método analítico fueron el peso de la muestra y el volumen de reactivo C. Posiblemente, al incrementar el peso de muestra no se logre una completa extracción de proteínas y puede disminuir el resultado final. El exceso del volumen de reactivo C, Folin-Ciocalteau, puede disminuir el pH y generar que el óptimo de absorbancia disminuya y alterar el resultado final.

Los factores menos sensibles (más robustos) fueron el volumen de Reactivo A, volumen de Reactivo B, tiempos de incubación y el tiempo de lectura.

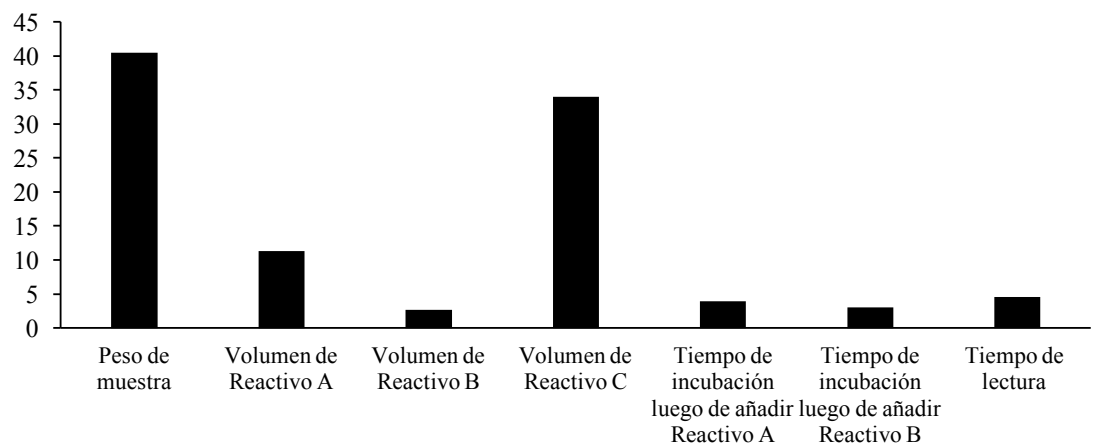

Figura 3. Efecto de los parámetros en el método analítico expresado en porcentaje

\section{CONCLUSIONES}

Se logró optimizar las condiciones de extracción e implementar la metodología analítica para la cuantificación del proteínas en la microalga Arthrospira platensis por espectrofotometría UV-Vis.

Las condiciones óptimas de extracción de proteínas en la microalga Arthrospira platensis fueron la concentración de $\mathrm{NaOH}=0,5 \mathrm{~N}$, tiempo $=60$ min y temperatura $=75^{\circ} \mathrm{C}$.

Los parámetros obtenidos de la implementación fueron: linealidad presentó un coeficiente de determinación de 0,9995 ; el límite de detección fue $2,36 \mu \mathrm{g} / \mathrm{mL}$ y el límite de cuantificación fue $7,86 \mu \mathrm{g} / \mathrm{mL}$; el porcentaje de recuperación fue $97,13 \%$; la precisión en condiciones de 
repetibilidad del método analítico presentó un $\mathrm{CV} \%$ de $1,79 \%$ y la robustez demostró que el método analítico es sensible al cambio en el peso de muestra y volumen de reactivo $\mathrm{C}$.

\section{AGRADECIMIENTO}

Los autores agradecen al laboratorio de Sala de Procesos por producir la muestra para nuestras pruebas y al Instituto del Mar del Perú por el apoyo brindado para la ejecución de este trabajo.

\section{BIBLIOGRAFÍA}

1. Priyadarshani I, Rath B. Commercial and industrial applications of microalgae - A review. J Algal Biomass Utln. 2012; 3(4): 89-100.

2. Rojas E, Ávila M, Parada G. Aplicación de estrategias nutricionales y su efecto en el crecimiento en el cultivo continuo de Spirulina (Arthrospira platensis). Lat Am J Aquat Res. 2012; 40(3): 763-771.

3. Moore JC, DeVries JW, Lipp M, Griffiths JC, Abernethy DR. Total Protein Methods and Their Potential Utility to Reduce the Risk of Food Protein Adulteration. Compr Rev Food Sci Food Saf. 2010; 9: 330-357.

4. AOAC International. Official Methods of Analysis of AOAC International. Rockville Maryland USA: AOAC International; 2016. ISBN: 0-935584-87-0.

5. Hartree EF. Determination of protein: a modification of the Lowry method that gives a linear photometric response. Anal Biochem. 1972; 48(2):422-7.

6. INDECOPI. Aprueban Guía para la validación de Métodos de Ensayo y las Directrices para la Implementación y Evaluación de métodos de Ensayo Sensoriales. [Internet]. El Peruano, 01 de Febrero de 2003, págs. 238363-238366. [consultado 15 jun 2017]. Disponible en: http://www.inacal.gob.pe/inacal/images/docs/acreditacion/requisitospara-solicitar-acreditacion/laborarios-ensayo-calibracion/guiaValidacion.pdf

7. Box G, Draper NR. Empirical model-building and response surfaces. Hoboken, New Jersey: Wiley; 1987. ISBN-10: 047471810339.

8. Arredondo B, Voltolina D. Métodos y herramientas analíticas en la evaluación de la biomasa microalgal. La Paz, Baja California Sur: Centro de Investigaciones Biológicas del Noroeste; 2007. ISBN: 968-5715-51-3.

9. AEFI. Validación de Métodos Analíticos. Barcelona: Asociación Española de Farmacéuticos en la Industria; 2001.

10. EPA. Method 6020B: Inductively Coupled Plasma - Mass Spectrometry. [Internet] SW846 Update V; 2014. $\neg[$ Consultado el 12 ago 2017]. Disponible en: https://www.epa. gov/sites/production/files/2015-12/documents/6020b.pdf

11. Srinivas CS, Puranik SB. HPLC Method Development and Validation for Dissolution of Vancomycin Hydrochloride from the Capsules. IJPPR. 2015; 4(2): 301-314.

12. FAO. Codex Alimentarius Commision Procedure Manual. Twenty-first edition. Roma: Food and Agriculture Organization of the United Nations; 2010. 